\title{
Valoración de la acogida de pacientes que inician terapia renal sustitutiva: Nivel de satisfacción
}

\author{
Ana Rebollo Rubio, Mª Eugenia Pons Raventos*, Mª José Macías López, Sandra Cabrera Azaña \\ Enfermeras. Hospital Regional Carlos Haya. UGC Nefrología, *Fresenius Medical Care Services Andalucía. \\ Málaga. España
}

\section{Resumen}

Introducción: En los últimos años han cobrado especial importancia aspectos tales como la calidad de vida, la salud percibida y la satisfacción del enfermo.

El objetivo de este estudio, es valorar el nivel de satisfacción de pacientes con enfermedad renal crónica con los cuidados recibidos, cuando inician por primera vez la terapia renal sustitutiva.

Material y métodos: Estudio descriptivo de corte transversal, en pacientes que inician por primera vez terapia renal sustitutiva. La muestra estuvo compuesta por 83 pacientes. Se recogieron variables sociodemográficas relacionadas con la enfermedad renal y seguimientos en consulta. Para valorar la satisfacción con los cuidados recibidos, a los pacientes se les entregó el cuestionario para medir Calidad de Vida Relacionada con la Salud (KDQOL-SF), específico para pacientes renales. Dentro de este cuestionario existe un apartado que mide la satisfacción con los cuidados recibidos.

Resultados: En el ítem SF23, el 62,7\% de la muestra consideró que la amabilidad del personal sanitario fue la mejor posible $(43,4 \%)$ o excelente $(19,3 \%)$. Por otro lado, en los ítems SF24a y $24 b$, el $51,8 \%$ de los pacientes opinan que el personal sanitario les anima a ser independientes y el $59 \%$ considera que les apoyan a hacer frente a la enfermedad. En el modelo de regresión ordinal utilizado, SF23 y SF24 obtiene mejores puntuaciones para diálisis peritoneal cuando se controlan el resto de variables demográficas, que no son significativas $(p<0,05)$.

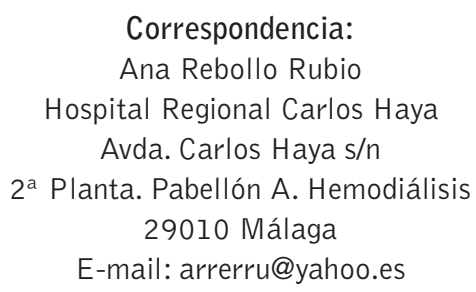

Conclusión: Valorando como muy positivos los resultados de satisfacción obtenidos hay que resaltar que resultan comparativamente mejores en la diálisis peritoneal.

PALABRAS CLAVE

- NIVEL DE SATISFACCIÓN

- HEMODIÁLISIS

- DIÁLISIS PERITONEAL

Assessment of reception of patients initiating renal replacement therapy: Level of satisfaction

\begin{abstract}
Introduction: In recent years have become very important issues such as quality of life, perceived health and patient satisfaction.
\end{abstract}

The aim of this study is to assess the level of satisfaction of patients with chronic kidney disease with care received when they first start renal replacement therapy.

Objective: A descriptive cross-sectional study in patients who start first renal replacement therapy. The sample consisted of 83 patients. Sociodemographic variables related to kidney disease and follow-on care clinics. To assess satisfaction with the care received, patients were given a questionnaire to measure Quality of Life Related to Health, specific for kidney patients. Within this section there is a questionnaire which measures satisfaction with the care received.

Results: In item SF23, 62.7\% of the sample felt that the friendliness of health workers was the best $(43.4 \%)$ or excellent (19.3\%). On the other hand, in SF24a and $24 \mathrm{~b}$ items, $51.8 \%$ of patients believe that health staff encourages them to be independent and $59 \%$ think it will support coping with illness. In the 
ordinal regression model used, SF23 and SF24 gets top scores for PD when other demographic variables that are not significant $(p<0.05)$ are controlled.

Conclusion: Considering as positive satisfaction results obtained should be highlighted that are comparatively better in peritoneal dialysis.

\section{KEYWORDS}

- SATISFACTION LEVEL

- HEMODIALYSIS

- PERITONEAL DIALYSIS

\section{Introducción}

La enfermedad renal crónica (ERC) causa un fuerte impacto en la vida de las personas que la padecen y de sus familiares. Es en el estadio $V$ de la enfermedad, momento en que se inicia la terapia renal sustitutiva (TRS), cuando esta enfermedad causa más estragos en el día a día de estos pacientes.

Actualmente, el ciudadano es el centro del sistema sanitario. Para los profesionales sanitarios y para los pacientes es importante que los cuidados que ofrecen los primeros sobre los segundos sean efectivos (ejerzan un efecto positivo en los niveles de salud), eficientes (ese efecto debería conseguirse a un coste asumible), aceptables (para el paciente que lo recibe y en opinión del conjunto de la profesión), accesibles (para el paciente en términos de distancia, espera, costes, etc.), han de ser valoradas como útiles por los propios pacientes y deben estar basados en evidencias (decisiones tomadas en base al conocimiento empírico y no en intuiciones) $)^{1,2}$.

Es por todo ello por lo que en los últimos años han cobrado importancia aspectos tales como la calidad de vida, la salud percibida y la satisfacción del enfermo. A pesar de esto, aún nos queda mucho por conocer de los factores que influyen en los mismos ${ }^{3,4}$. Además, el nivel de satisfacción del paciente con las atenciones y tratamiento que recibe es un resultado mensurable de la intervención clínica ${ }^{5}$.

Por otro lado parece quedar inherente al concepto de satisfacción el concepto de expectativas y es que el grado de satisfacción no depende solo de la prestación de los servicios, sino también de la solución de sus expectativas, ya que el paciente tendrá más satisfacción cuando dichos servicios reúnan o sobrepasen las características que esperaban 6 .
Es la ERC una enfermedad que utiliza un gran consumo de recursos sanitarios, $y$ una enfermedad en la que el paciente y el personal sanitario que lo atiende tienen una estrecha relación al verse al menos 3 veces por semana durante 4 horas en el caso de la hemodiálisis (HD) y durante muchas horas dedicadas al entrenamiento y posterior seguimiento en el caso de la diálisis peritoneal (DP). Por esto hemos creído importante saber cuál es la percepción del paciente con respecto al personal que lo atiende en el momento de iniciar la TRS, cómo les valora y cómo cree que son los cuidados recibidos por parte de este personal con el que mantendrá una estrecha relación durante el curso de su enfermedad, hasta que reciba un trasplante renal, 0 en caso de no ser posible esto el resto de su vida.

El objetivo de este estudio ha sido valorar la satisfacción con los cuidados recibidos en pacientes con ERCA (enfermedad renal crónica avanzada) en términos de amabilidad, interés y apoyo mostrado por el personal de enfermería y nefrólogos de la unidad donde inician por primera vez TRS medido en el primer mes de su entrada a diálisis.

\section{Material y método}

Estudio descriptivo de corte transversal, en pacientes que inician por primera vez TRS en los todos los centros de la provincia de Málaga, durante el periodo que abarca desde el 1 abril de 2013 al 31 de marzo de 2014 y hayan dado su consentimiento para participar en dicho estudio. Los criterios de exclusión fueron: incapacidad física o intelectual para contestar al cuestionario y trasplante renal previo a iniciar TRS. Los centros participantes fueron: Hospital Regional Universitario Carlos Haya, Centro de Diálisis Málaga - El Cónsul FMC, Centro de Diálisis Málaga - Ciudad Jardín FMC, Clínica B. Braun Málaga, Centro de Diálisis de Málaga (Diaverum), Centro de Diálisis La Axarquía, Centro de Diálisis Torremolinos, Clínica de Diálisis Antequera FMC, Hospital Quirón Marbella, Hospital Costa del Sol (Marbella), Centro de Diálisis Estepona y Hospital Serranía de Ronda. Finalmente fueron reclutados 83 pacientes que iniciaron la TRS en cualquiera de sus modalidades.

Las variables sociodemográficas que se recogieron fueron: edad, sexo, estado civil, situación laboral y nivel educativo. Las variables que se obtuvieron referentes a la ERC fueron: distancia al centro nefrológico de referencia, etiología de la ERC, índice de comorbilidad de Charlson (ICC), presencia de algún familiar en TRS, modalidad de TRS, acceso vascular (cuando la TRS fue hemodiálisis). Para valorar la satisfacción con los cuidados recibidos, a los pacientes se les entregó el cuestionario específico para pacientes renales que mide Calidad de Vida Relacionada con la Salud 
(Kidney Disease Quality of Life - Short Form). La validación del Kidney Disease Quality of Life - Short Form (KDQOL-SF) para la población española en una muestra de 306 pacientes procedentes de 35 centros de diálisis, indicó coeficientes alfa de Cronbach superiores a 0.65 para todas las escalas, excepto para el trabajo (alfa $=0.39$ ) y calidad de la interacción social (alfa=0.61), así como correlaciones más significativas con capacidad funcional y correlaciones negativas significativas con comorbilidad y diabetes en la mayoría de las escalas ${ }^{7-10}$. Este instrumento dispone de una versión española validada. Dentro de este cuestionario existe un apartado que mide la satisfacción con los cuidados recibidos, estos son los ítems KDQOLSF23, 24a y 24b. El ítem SF23 valora la amabilidad y trato del personal sanitario en una escala Likert directa de 1 a 7. El ítem SF24 valora el interés del personal por aumentar el afrontamiento del paciente en una escala Likert indirecta del 1 al 5, con un apartado a en relación al fomento de la independencia y un apartado $b$ en relación al fomento del manejo de la enfermedad. Finalmente, también se registró si el paciente había llevado a cabo un seguimiento reglado en la etapa prediálisis por nefrólogo y enfermera. Además de registrar el tiempo de seguimiento y el estadio de ERC en el inicio el seguimiento.

Durante una entrevista con el paciente, se le informó del objetivo del estudio y tras la firma de consentimiento informado se recogieron las variables sociodemográficas. Se revisó la historia clínica de los pacientes participantes para extraer los datos referentes a las consultas de ERC, meses de seguimiento, estadio, etc. En ésta entrevista se les entrega el cuestionario KDQOL-SF, siendo la forma de administración "autoadministrado".

En el análisis estadístico, las variables categóricas han sido descritas con frecuencia y porcentaje. Para las variables numéricas utilizamos media \pm desviación típica (DT), si siguen una distribución normal, si no es así, mediana y rango intercuartílico. Hemos estudiado la normalidad de los datos aplicando la prueba de Kolmogorov - Smirnov. En el análisis univariante, cuando ambas variables son categóricas hemos contrastado su grado de independencia con la prueba Chi-Cuadrado $\left(X^{2}\right)$. También hemos empleado los estadísticos d de Somers, Gamma y Tau de Kendall para el análisis de correlaciones entre variables. Estos estadísticos se basan en el núme- ro de concordancias y discordancias que aparecen al comparar las puntuaciones asignadas. Las diferencias se considerarán estadísticamente significativas para $\mathrm{p}<0.05$ con un intervalo de confianza (IC) del $95 \%$. Para el análisis de los datos se utilizará el paquete estadístico SPSS Statistics Versión 21.

\section{Resultados}

Las características basales de la población de estudio se muestran en la tabla 1.

Tabla 1. Características basales de la población de estudio.

\begin{tabular}{|c|c|c|}
\hline Edad & 59,9 años $\pm 14,34$ & \\
\hline \multirow[t]{2}{*}{ Sexo } & Hombre $[n(\%)]$ & $n=59(71,1 \%)$ \\
\hline & Mujer [n (\%)] & $n=24(28,9 \%)$ \\
\hline \multirow[t]{4}{*}{ Estado Civil } & Soltero/a [n (\%)] & $n=4(5,8 \%)$ \\
\hline & Casado/a o en pareja $[\mathrm{n}(\%)]$ & $n=57(82,6 \%)$ \\
\hline & Viudo/a $[n(\%)]$ & $n=4(5,8 \%)$ \\
\hline & Divorciado/a o separado/a [n (\%)] & $n=4(5,8 \%)$ \\
\hline \multirow[t]{4}{*}{ Situación laboral } & Activo/a [n (\%)] & $n=7(10,3 \%)$ \\
\hline & Invalidez T/P [n (\%)] & $n=12(17,6 \%)$ \\
\hline & Jubilado/a [n (\%)] & $n=37(54,4 \%)$ \\
\hline & En paro o ama de casa $[n(\%)]$ & $n=12(17,6 \%)$ \\
\hline \multirow[t]{4}{*}{ Nivel educativo } & Sin estudios $[n(\%)]$ & $\mathrm{n}=10(14,5 \%)$ \\
\hline & Primarios [n (\%)] & $n=41(59,4 \%)$ \\
\hline & Secundarios $[n(\%)]$ & $n=8(11,6 \%)$ \\
\hline & Universitarios [n (\%)] & $\mathrm{n}=10(14,5 \%)$ \\
\hline \multicolumn{3}{|l|}{ Distancia al centro } \\
\hline \multirow[t]{4}{*}{ de TRS ${ }^{1}$} & $<10 \mathrm{Km}$ & $n=32(46,4 \%)$ \\
\hline & Entre 11 y $30 \mathrm{Km}$ & $\mathrm{n}=10(14,5 \%)$ \\
\hline & Entre 31 y $50 \mathrm{Km}$ & $n=8(11,6 \%)$ \\
\hline & $>50 \mathrm{Km}$ & $\mathrm{n}=19(27,5 \%)$ \\
\hline \multirow[t]{5}{*}{ Etiología de la ERC ${ }^{2}$} & Glomerulonefritis [n (\%)] & $\mathrm{n}=14(17,5 \%)$ \\
\hline & Diabetes $[n(\%)]$ & $n=19(23,8 \%)$ \\
\hline & $\operatorname{Vascular}[n(\%)]$ & $\mathrm{n}=18(22,5 \%)$ \\
\hline & Enfermedad poliquística [n (\%)] & $\mathrm{n}=9(11,3 \%)$ \\
\hline & Otros $[n(\%)]$ & $n=20(25 \%)$ \\
\hline \multirow[t]{2}{*}{ Familiar en TRS ${ }^{1}$} & Sí [n (\%)] & $n=11(16,4 \%)$ \\
\hline & No $[n(\%)]$ & $n=56(83,6 \%)$ \\
\hline \multirow[t]{2}{*}{ Modalidad de TRS ${ }^{1}$} & Hemodiálisis [n (\%)] & $n=63(77,8 \%)$ \\
\hline & Diálisis Peritoneal $[n(\%)]$ & $\mathrm{n}=18(22,2 \%)$ \\
\hline \multirow[t]{3}{*}{ Acceso vascular } & $\mathrm{FAV}^{3}[\mathrm{n}(\%)]$ & $n=31(63,3 \%)$ \\
\hline & Catéter venoso permanente $[\mathrm{n}(\%)]$ & $n=15(30,6 \%)$ \\
\hline & Catéter venoso transitorio [n (\%)] & $n=3(6,1 \%)$ \\
\hline Índice de Charlson & $4(2-5)$ & \\
\hline \multirow[t]{2}{*}{ Seguimiento Nefrólogo } & Sí $[n(\%)]$ & $n=69(83,5 \%)$ \\
\hline & No $[n(\%)]$ & $n=14(16,5 \%)$ \\
\hline \multirow[t]{2}{*}{ Seguimiento Enfermera } & Sí [n (\%)] & $\mathrm{n}=31(33,7 \%)$ \\
\hline & No $[n(\%)]$ & $n=52(66,3 \%)$ \\
\hline
\end{tabular}

Las variables categóricas se han expresado con frecuencia y porcentaje. Las variables numéricas que siguen una distribución normal se han expresado con media \pm desviación típica y en caso contrario con mediana (percentil 25 - percentil 75).

${ }^{1}$ Tratamiento renal sustitutivo. ${ }^{2}$ Enfermedad renal crónica. ${ }^{3}$ Fístula arteriovenosa. 
Pese a que la etiología dominante es de instauración progresiva y la edad de los pacientes oscila entre los 50 y los 70 años en más del $50 \%$ de la muestra, tan sólo un $33,7 \%$ llevaron un seguimiento reglado en consulta de enfermería ERCA en la etapa prediálisis, frente a un $83,5 \%$ que tuvieron seguimiento nefrológico.

Los pacientes fueron derivados a consulta de enfermería en un estadio ERC 4 o 5 en el $100 \%$ de los casos con una media de seguimiento de 10,88 meses (DT 12,14). El 63,3\% de los pacientes portaban una fístula arteriovenosa (FAV) como acceso vascular inicial para la HD. Realizando inferencia estadística resultó significativo que aquellos pacientes que tienen ocasión de pasar por la consulta de enfermería comenzaron TRS con una FAV realizada (Gamma y $d$ de Sommers $p<0,05)$. No hubo relación estadística sin embargo entre el método de TRS utilizado y el paso previo por la consulta de enfermería (tabla 2 ).
En la figura 2, mostramos los resultados obtenidos con respecto al ítem SF-24a del cuestionario KDQOL-SF tras el enunciado, "¿En qué medida son ciertas o falsas cada una de las siguientes afirmaciones? El personal de diálisis me anima a ser todo lo independiente posible."

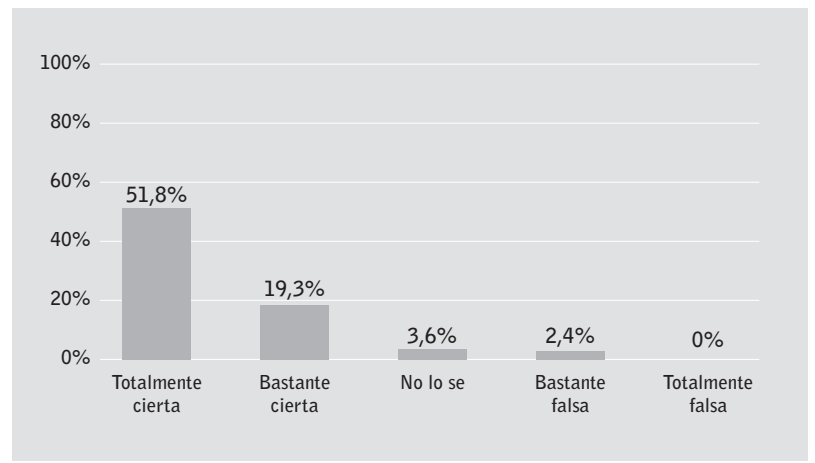

Figura 2. Resultados obtenidos en el ítem 24a del cuestionario de Calidad de Vida Relacionada con la Salud (KDQOL-SF).
Tabla 2. Distribuciones observadas del acceso vascular y de la modalidad de TRS al inicio del tratamiento, en base a los seguimientos en la consulta de enfermería.

\begin{tabular}{|c|c|c|c|c|c|c|}
\hline & & \multicolumn{4}{|c|}{$\begin{array}{c}\text { ¿Seguimiento en la consulta ERC }{ }^{1} \\
\text { Enfermería? }\end{array}$} & \multirow{3}{*}{$\begin{array}{c}\text { Significación } \\
\text { aproximada } \\
\text { (Gamma, d de Sommers } \\
\text { y Tau de Kendall) }\end{array}$} \\
\hline & & \multicolumn{2}{|c|}{ No } & \multicolumn{2}{|r|}{$\mathrm{Si}$} & \\
\hline & & $\mathrm{n}$ & $\%$ & $\mathrm{n}$ & $\%$ & \\
\hline \multirow{3}{*}{ Acceso vascular } & $\mathrm{FAV}^{2}$ & 17 & $51,5 \%$ & 13 & $86,7 \%$ & \multirow{3}{*}{$P=0,004$} \\
\hline & CVC $^{3}$ permanente & 13 & $39,4 \%$ & 2 & $13,3 \%$ & \\
\hline & $\mathrm{CVC}^{3}$ temporal & 3 & $9,1 \%$ & 0 & $0,0 \%$ & \\
\hline \multirow{2}{*}{ Modalidad de TRS ${ }^{4}$} & Hemodiálisis & 43 & $84,3 \%$ & 19 & $67,9 \%$ & \multirow{2}{*}{$P=0,108$} \\
\hline & Diálisis peritoneal & 8 & $15,7 \%$ & 9 & $32,1 \%$ & \\
\hline
\end{tabular}

${ }^{1}$ Enfermedad Renal Crónica. ${ }^{2}$ Fístula arteriovenosa. ${ }^{3}$ Catéter venoso central. ${ }^{4}$ Terapia Sustitutiva Renal.
Finalmente en la figura 3, presentamos los resultados obtenidos con respecto al ítem SF-24b del cuestionario KDQOL-SF tras el enunciado, "¿En qué medida son ciertas o falsas cada una de las siguientes afirmaciones? El personal de diálisis me apoya para hacer frente a mi enfermedad del riñón."
En la figura 1, podemos observar los resultados obtenidos con respecto al ítem SF-23 del cuestionario KDQOL-SF tras el enunciado, "Piense en la atención que recibe en la diálisis, según su grado de satisfacción, la amabilidad y el interés que muestran hacia usted como persona".

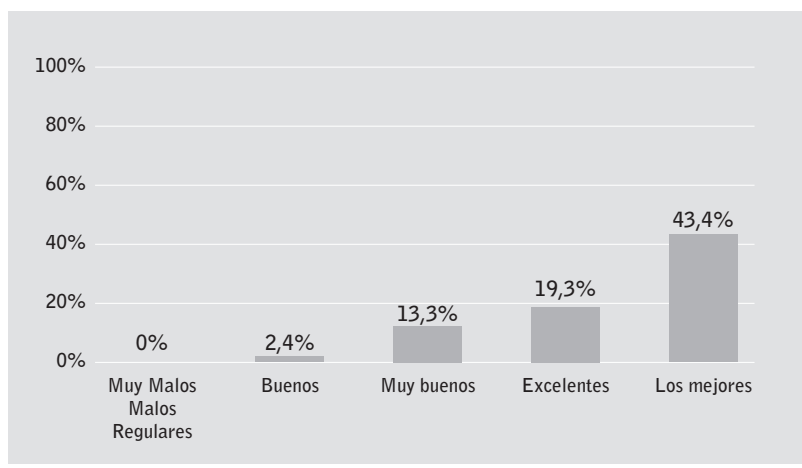

Figura 1. Resultados obtenidos en el ítem 23 del cuestionario de Calidad de Vida Relacionada con la Salud (KDQOL-SF).

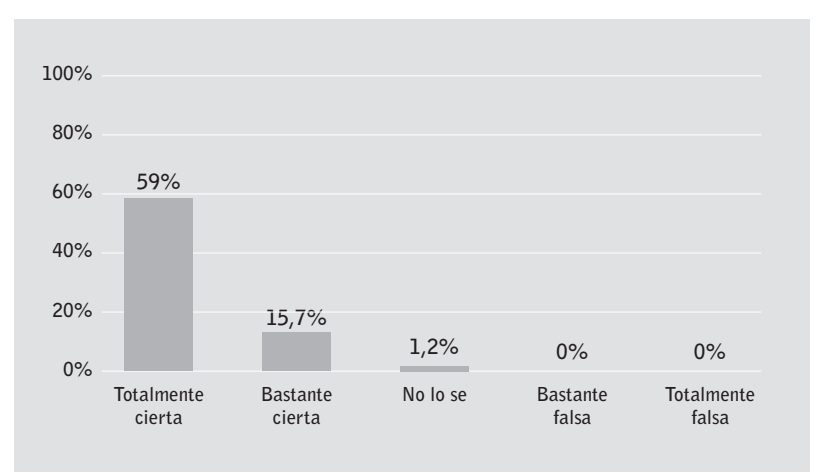

Figura 3. Resultados obtenidos en el ítem $24 b$ del cuestionario de Calidad de Vida Relacionada con la Salud (KDQOL-SF).

En el modelo de regresión ordinal utilizado, SF23 y SF24 obtiene mejores puntuaciones para DP cuando se controlan el resto de variables demográficas, que no son significativas (Gamma, d de Sommers y Tau de Kendall $p<0,05$ ). 


\section{Discusión}

Un estudio multicéntrico sobre calidad asistencial percibida por los pacientes en programa de hemodiálisis de Tarragona que entrevistó a 148 pacientes con el cuestionario SERVQHOS, muestra que los aspectos mejor puntuados por los pacientes sobre la calidad asistencial percibida fueron, por orden: la amabilidad del personal, la preparación del personal, la disposición del personal para ayudar y solucionar problemas, el trato personalizado y el interés del personal de enfermería. Por otro lado constataron que los aspectos más importantes para el paciente son, por orden: la amabilidad del personal, la disposición del personal para ayudar, la preparación del personal, el interés para solucionar problemas, la confianza y seguridad que el personal sanitario trasmite, el trato personalizado, y el interés del personal de enfermería ${ }^{11}$.

Otro estudio de Santos y cols. en nuestro país, encuentra que la satisfacción general de los pacientes es alta y que la profesionalidad del personal sanitario es valorada positivamente. Estos autores no encuentran relación entre la satisfacción y las variables sociodemográficas ${ }^{12}$. Estos resultados concuerdan con los obtenidos en nuestro estudio, no habiendo encontrado asociación entre las variables sociodemográficas y la satisfacción con los cuidados recibidos por parte del personal que los atiende en los momentos que el paciente inicia TRS. Aunque no existen diferencias significativas en ninguno de los tres ítems según la técnica de TRS elegida, parece que en los pacientes que se decantan por la técnica de DP, esa satisfacción percibida es mayor, quedando esto explicado probablemente por el tiempo que el paciente pasa con la enfermera/nefrólogo en la etapa de entrenamiento y el vínculo más estrecho que se establece entre ellos. En nuestro estudio queda patente que la percepción que el paciente tiene sobre la atención recibida al inicio de la TRS, es muy positiva. Un $62,7 \%$ de los pacientes perciben que la amabilidad y el interés del personal que le atiende en diálisis son los mejores o excelentes. Un $71,1 \%$ de la muestra contesta que la afirmación de que el personal de diálisis le anima a ser lo más independiente posible es totalmente cierta o bastante cierta y un $74,7 \%$ de los pacientes entrevistados considera totalmente cierta o bastante cierta la afirmación de que el personal que le atiende en diálisis le apoya para hacer frente a su enfermedad del riñón. Destacamos también que ningún paciente ha elegido la respuesta más negativa de la escala Likert en ninguna de las tres preguntas.

\section{Conclusión}

El propósito de este estudio ha sido valorar la satisfacción con los cuidados recibidos por parte del personal nefrológico (nefrólogos y personal de enfermería) que atienden a los pacientes que inician TRS en sus dos modalidades (HD y DP). Los pacientes se sienten apoyados en su enfermedad por el personal de nefrología que los atiende. En la muestra de pacientes analizados, hemos constatado muy positivamente la satisfacción con los cuidados recibidos en el momento de iniciar la TRS.

\section{Agradecimientos}

Nuestro más sincero agradecimiento a todos los pacientes que muy amablemente se han ofrecido a participar en nuestro estudio en estos momentos tan duros para ellos.

\section{Conflicto de intereses}

Los autores declaran no tener ningún conflicto de intereses.

Recibido: 20 noviembre 2014

Revisado: 10 diciembre 2014

Modificado: 12 enero 2015

Aceptado: 15 enero 2015

\section{Bibliografía}

1. Elley CR, Kerse N,Arroll B, Robinson E. Effectiveness of counselling patients on physical activity in general practice: cluster randomised controlled trial. BMJ 2003; 326(7393):793.

2. Britten N. Patients' expectations of consultations. BM 2004; 328(7437):416-7.

3. Mira JJ, Buil JA, Aranaz J, Vitaller J, Lorenzo $S$, Ignacio $E$, et al. ¿Qué opinan los pacientes de los hospitales públicos? Análisis de los niveles de calidad percibida en cinco hospitales. Gac Sanit 2000;14(4):291-3.

4. Arenas MD, Moreno E, Reig A, Millán I, Egea JJ, Amoedo ML, et al. Evaluación de la calidad de vida relacionada con la salud mediante las láminas CoopWonca en una población de hemodiálisis. Nefrología 2004;25(5):470-9. 
5. Mira JJ, Aranaz J. La satisfacción del paciente como una medida del resultado de la atención sanitaria. Med Clínica 2000;111(Suppl 3):26-33.

6. Thompson AG, Suñol R. Expectations as determinants of patient satisfaction: concepts, theory and evidence. Int J Qual Health Care 1995; 7(2):127-41.

7. Vázquez I, Valderrábano F, Fort I, Jofré R, LópezGómez JM, Moreno F, et al. Diferencias en la calidad de vida relacionada con la salud entre hombres y mujeres en tratamiento en hemodiálisis. Nefrología 2004;24(2):167-78.

8. García F, López K, Alvaro F, L P, Alonso J. Spanish validation of the short form of the Kidney Disease Quality of Life Instrument. Qual Life Res 1999.

9. García F, López K, De Álvaro, Álvarez UF, Alonso $J$, en representación del grupo CALVIDIA. Salud percibida en pacientes que comienzan tratamiento renal sustitutivo: validación preliminar de la versión española del KDQOL-SF. Nefrología 1998; 18 Suppl 3: 66.
10. Hays RD, Kallich J, Mapes DL, Coons SJ, Amin N, Carter WB. Kidney Disease Quality of Life Short Form (KDQOL-SF) Version 1.2: a manual for use and scoring. St Mónica, CA: RAND; 1997.

11. Guinovart EA, Baterman FM. Estudio multicéntrico sobre la calidad asistencial percibida por los pacientes en programa de hemodiálisis de Tarragona. Soc Esp Enferm Nefrol 2011;14(3):172-80.

12. Cerro P, Matani V, Santos AC. ¿Qué opinan los pacientes de diálisis del Hospital Universitario Nuestra Señora de la Candelaria sobre los servicios recibidos? Valoración de la calidad percibida. NURE Inv [Revista en Internet] 2008;5(33). Disponible en: http://www.nureinvestigation.es 